\title{
SOLUTION OF LINEAR DIFFERENCE AND DIFFERENTIAL EQUATIONS
}

BY J. F. TRAUB

\author{
Communicated by A. S. Householder, December 30, 1964
}

1. Introduction. The solution of an $n$th order inhomogeneous difference equation as an explicit function of the starting conditions (without using determinants) has been given by Traub [2], the proof based on a division algebra for sequences. In this note we prove two identities, (1) and (6), which may be used to provide a direct proof of the formulas, (13) and (14), for the solution of inhomogeneous difference or differential equations with constant coefficients. No transforms are required. These solutions are in a form which is felicitous for a number of applications.

2. Two identities. Let $P(t)$ be a monic polynomial

$$
P(t)=\sum_{j=0}^{n} a_{n-j} t^{j}, \quad a_{0}=1,
$$

with complex coefficients and with distinct zeros $\rho_{1}, \rho_{2}, \cdots, \rho_{n}$. (The extension to the case of confluent zeros will be treated elsewhere.) Let $\lambda$ be a nonnegative integer. Then

$$
t^{\lambda}=P(t) \sum_{i=1}^{n} \frac{\rho_{i}^{\lambda}}{\left(t-\rho_{i}\right) P^{\prime}\left(\rho_{i}\right)}+P(t) \sum_{j=0}^{\lambda-1} t^{\lambda-1-j} \Omega(j),
$$

where

$$
\Omega(j)=\sum_{i=1}^{n} \frac{\rho_{i}^{j}}{P^{\prime}\left(\rho_{i}\right)} .
$$

We remark that $\Omega(j)$, a symmetric polynomial in the $\rho_{i}$, may be obtained by translation from Wronski's aleph function [1],

$$
\omega(j)=\sum_{i=1}^{n} \frac{\rho_{i}^{n-1+j}}{P^{\prime}\left(\rho_{i}\right)} .
$$

It is well known that

$$
\Omega(j)=\delta_{n-1, j}, \quad j=0,1, \cdots, n-1 .
$$


To prove (1), we observe that, by division,

$$
t^{\lambda}=G(\lambda, t)+P(t) Q(\lambda, t)
$$

where $G(\lambda, t)$ is a polynomial of degree $n-1$ and $Q(\lambda, t)$ is a polynomial of degree $\lambda-n$ if $\lambda \geqq n$, and is identically zero otherwise. By Lagrange's interpolation formula,

$$
G(\lambda, t)=\sum_{i=1}^{n} \frac{P(t) \rho_{i}^{\lambda}}{P^{\prime}\left(\rho_{i}\right)\left(t-\rho_{i}\right)} .
$$

Let $E$ be the translation operator, $E f(\lambda)=f(\lambda+1)$. Then

$$
(t-E) G(\lambda, t)=P(t) \Omega(\lambda) .
$$

Applying $t-E$ to (3) shows that $Q(\lambda, t)$ satisfies the first-order difference equation,

$$
0=\Omega(\lambda)+(t-E) Q(\lambda, t)
$$

Since $Q(0, t)=0$,

$$
Q(\lambda, t)=\sum_{j=0}^{\lambda-1} t^{\lambda-1-j} \Omega(j)
$$

Substitution of (4) and (5) into (3) yields the result.

We next show that

$$
e^{x t}=P(t) \sum_{i=1}^{n} \frac{e^{\rho_{i} x}}{P^{\prime}\left(\rho_{i}\right)\left(t-\rho_{i}\right)}+P(t) \int_{0}^{x} V(u) e^{t(x-u)} d u,
$$

where

$$
V(u)=\sum_{i=1}^{n} \frac{e^{\rho_{i} u}}{P^{\prime}\left(\rho_{i}\right)} .
$$

(Observe that $V^{(j)}(0)=\Omega(j)$.)

To prove (6) we use the polynomial interpolation formula with remainder to obtain

$$
\begin{aligned}
e^{x t} & =H(x, t)+P(t) R(x, t), \\
H(x, t) & =\sum_{i=1}^{n} \frac{P(t) e^{\rho_{i} x}}{\left(t-\rho_{i}\right) P^{\prime}\left(\rho_{i}\right)}
\end{aligned}
$$

Although $R(x, t)$ could be expressed as an $n$th divided difference, and, hence, as an iterated integral, we prefer to obtain it directly. Let $D$ be the operator of differentiation with respect to $x$. Then

$$
(t-D) H(x, t)=P(t) V(x) \text {. }
$$


Apply $t-D$ to (8) to show that

$$
0=V(x)+(t-D) R(x, t) .
$$

Setting $x=0$ in (8) shows that $R(0, t)=0$. Hence,

$$
R(x, t)=\int_{0}^{x} V(u) e^{t(x-u)} d u .
$$

Substitution of (9) and (10) into (8) yields the result.

One may also verify (1) or (6) by performing the summation or integration of the second term on the right.

3. Solution of linear equations. For our application it is convenient to perform the division $P(t) /\left(t-\rho_{i}\right)$ in (1) and (6). Let

$$
\frac{P(t)}{t-\rho_{i}}=\sum_{k=0}^{n-1} A_{k}\left(\rho_{i}\right) t^{n-1-k}
$$

where the $k$ th degree polynomial $A_{k}(t)$ satisfies the "nesting" recursion

$$
A_{k}(t)=t A_{k-1}(t)+a_{k}, \quad k=0,1, \cdots, n, \quad A_{-1}(t)=0,
$$

and is explicitly given by

$$
A_{k}(t)=\sum_{r=0}^{k} a_{k-r} t^{r}
$$

Then (1) and (6) become

$$
\begin{aligned}
t^{\lambda} & =\sum_{k=0}^{n-1} t^{n-1-k} \sum_{i=1}^{n} \frac{\rho_{i}^{\lambda} A_{k}\left(\rho_{i}\right)}{P^{\prime}\left(\rho_{i}\right)}+P(t) \sum_{j=0}^{\lambda-1} t^{\lambda-1-j} \Omega(j), \\
e^{x t} & =\sum_{k=0}^{n-1} t^{n-1-k} \sum_{i=1}^{n} \frac{e^{\rho_{i} x} A_{k}\left(\rho_{i}\right)}{P^{\prime}\left(\rho_{i}\right)}+P(t) \int_{0}^{x} V(u) e^{t(x-u)} d u .
\end{aligned}
$$

We wish to solve the difference equation

$$
P(E) f(\lambda)=g(\lambda),
$$

with the starting conditions

$$
E^{j} f(0)=y_{j}, \quad j=0,1, \cdots, n-1 .
$$

Taking $t=E$ in (11) and observing that

$$
f(\lambda)=E^{\lambda} f(0), \quad P(E) f(0)=g(0),
$$

yields the result 


$$
f(\lambda)=\sum_{k=0}^{n-1} y_{n-1-k} \sum_{i=1}^{n} \frac{\rho_{i}^{\lambda} A_{k}\left(\rho_{i}\right)}{P^{\prime}\left(\rho_{i}\right)}+\sum_{j=0}^{\lambda-1} g(\lambda-1-j) \Omega(j),
$$

with $\Omega(j)$ defined by (2).

We turn to the differential equation

$$
P(D) f(x)=g(x),
$$

with initial conditions

$$
D^{j} f(0)=y_{0}^{(j)}, \quad j=0,1, \cdots, n-1 .
$$

Taking $t=D$ in (12) and observing that

$$
f(x)=e^{x D} f(0), \quad P(D) f(0)=g(0), \quad e^{(x-u) D} g(0)=g(x-u),
$$

yields the result

$$
f(x)=\sum_{k=0}^{n-1} y_{0}^{(n-1-k)} \sum_{i=1}^{n} \frac{e^{\rho_{i} x} A_{k}\left(\rho_{i}\right)}{P^{\prime}\left(\rho_{i}\right)}+\int_{0}^{x} g(x-u) V(u) d u,
$$

with $V(u)$ defined by (7).

\section{REFERENCES}

1. Encyklop. d. Math. Wissensch., Vol. I, Part I, Sect. 3b. Rationale Funktionen der Wurzeln, pp. 450-479.

2. J. F. Traub, Generalized sequences with applications to the discrete calculus, Math. Comp. 19 (1965), 177-200.

Bell Telephone laboratories, Incorporated, Murray Hill, New Jersey 